\title{
Should ICSI be recommended routinely in patients with four or fewer oocytes retrieved?
}

\author{
Martha Luna • Catherine Bigelow • Marlena Duke • \\ Jane Ruman • Benjamin Sandler • Lawrence Grunfeld • \\ Alan B. Copperman
}

Received: 15 April 2011 / Accepted: 11 July 2011 /Published online: 27 July 2011

(C) Springer Science+Business Media, LLC 2011

\begin{abstract}
Purpose To determine if patients with a low response to controlled ovarian hyperstimulation during IVF benefit from intracytoplasmic sperm injection (ICSI)

Methods Retrospective analysis of 350 IVF cycles in which four or fewer oocytes were retrieved. Severe male factor cases were excluded from analysis. Conventional insemination (CI) and ICSI were compared, with primary outcome measures of fertilization rate, implantation rate, clinical pregnancy rate per embryo transfer, and pregnancy loss rate.

Result(s) Fertilization rates per oocyte retrieved for $\mathrm{CI}$ and ICSI were comparable (51.5\% vs. $51.8 \%)$. Parallel implantation rates ( $22 \%$ vs. $25 \%)$, clinical pregnancy rates $(32.8 \%$ vs. $33.3 \%)$, and loss rates ( $26.7 \%$ vs. $39.5 \%)$ were also noted. No difference in cancelled cycles was reported.

Conclusion(s) Our results demonstrate that in the presence of normal semen parameters, low egg number is not an indication to perform ICSI.
\end{abstract}

Capsule In the presence of normal semen parameters, low response to ovarian hyperstimulation is not an indication to perform ICSI over conventional insemination.

M. Luna $\cdot$ C. Bigelow $\cdot$ M. Duke $\cdot$ J. Ruman $\cdot$ B. Sandler $\cdot$

L. Grunfeld $\cdot$ A. B. Copperman

Reproductive Medicine Associates of New York,

New York, NY, USA

M. Luna $\cdot$ C. Bigelow $\cdot$ M. Duke $\cdot$ J. Ruman $\cdot$ B. Sandler $\cdot$

L. Grunfeld · A. B. Copperman

Department of Obstetrics and Gynecology and Reproductive

Science - Division of Reproductive Endocrinology and Infertility,

Mount Sinai School of Medicine,

New York, NY, USA

A. B. Copperman $(\bowtie)$

635 Madison Ave, 10th Floor,

New York, NY 10022, USA

e-mail: acopperman@rmaofny.com
Keywords IVF · ICSI · Poor response · Oocyte retrieval

\section{Introduction}

Male factor infertility is a well-established indication for intracytoplasmic sperm injection (ICSI) [1-3]. Assisting fertilization allows bypass of the zona pellucida with a single spermatozoon [4]. Since the report of the first human pregnancies achieved by this technique, ICSI has been applied extensively worldwide. There is still conflict, however, regarding the use of ICSI in couples with nonmale factor infertility or unexplained infertility.

ICSI has been associated with high fertilization and pregnancy rates regardless of sperm concentration, motility or morphology [5-7]. The rate of ICSI procedures in cycles without male factor infertility has increased progressively during the last 7 years, comprising $39 \%$ of all ICSI cases in 2000 and rising to greater than $50 \%$ of cases in 2006 . It seems as though ICSI has been universally promoted and is increasingly replacing conventional insemination due to its many benefits and lack of definable drawbacks [8].

Indications for performing ICSI include male factor infertility, history of failed or low fertilization rate, unexplained infertility, couples who lack a history of pregnancies, and immunological infertility [6, 7, 9-11]. A subset of patients in whom the risk-benefit of performing ICSI is unclear is low responding patients. An association between lower number of retrieved oocytes and lower fertilization rates and IVF outcome has been described [12, 13]. Additionally, fertilization failure is a common finding when less than 3 oocytes are retrieved [14] The high fertilization rates, small numbers of fertilization failures, and lack of apparent long term drawbacks make ICSI an appealing solution for IVF $[15,16]$. Hence, it has 
been proposed that ICSI could improve fertilization rates and the likelihood of successful embryo transfer when the number of recovered oocytes is low [17]. We decided to engage in the comparison of outcome of two fertilization techniques in patients who demonstrate a low response to gonadotropin stimulation, without any indication for ICSI.

\section{Materials \& methods}

Patient data

A total of 350 IVF cycles with a low response to stimulation at Reproductive Medicine Associates of New York were retrospectively analyzed. The database was searched for IVF cycles performed from September 2002 and March 2009 based on number of retrieved oocytes and insemination technique. Pertinent charts were reviewed for cycle data and outcomes. All cycles in which four or fewer oocytes were retrieved following controlled ovarian hyperstimulation were included. Cases with severe male factor infertility were excluded from this analysis. ICSI was performed based on physician preference due solely to low oocyte yield. Institutional Review Board approval was obtained for the performance of this retrospective analysis.

\section{Controlled ovarian hyperstimulation and oocyte retrieval}

Controlled ovarian stimulation was achieved using GnRH antagonist, Down-Regulation, Microflare and Estrogen Priming protocols. Patients were serially monitored for estradiol and progesterone levels, as well as count and size of follicles using transvaginal ultrasound in the office. Patients were administered human chorionic gonadotropin (hCG) injection with the presence of two $>17 \mathrm{~mm}$ follicles. Oocyte retrieval was performed under conscious sedation using a transvaginal ultrasound probe and needle aspiration $36 \mathrm{~h}$ after administration of hCG.

Gamete preparation, fertilization and embryo culture

Fresh ejaculated semen samples were provided on the day of vaginal oocyte retrieval (VOR). These samples were evaluated for sperm count; $>20$ million motile sperm were considered adequate for CI. Semen samples were washed and processed for CI or ICSI. Conventional insemination was performed 4-6 h after VOR. One to three oocytes with surrounding cumulus com- plex were placed in $50 \mu \mathrm{L}$ drops of media with approximately 50,000 motile sperm. For cases undergoing ICSI, oocytes were denuded of the cumulus complex 3-4 h after VOR using hyaluronidase. Oocytes were assessed for maturity by the presence of an expelled first polar body. Sperm were selected based on morphology and motility, the mitochondrial tail was disrupted with the injection pipette, and the single sperm was injected into the 3 o'clock position of the mature oocyte 5-6 $\mathrm{h}$ after VOR. All metaphase I oocytes were discarded.

Assessment of embryo quality for transfer

Fertilization was confirmed $16-18 \mathrm{~h}$ following CI or ICSI by the presence of two pronuclei in the zygote. Oocytes that failed to fertilize after conventional insemination were assessed for maturity. Conventionally inseminated oocytes that demonstrated one pronucleus were maintained in culture and evaluated the following day. Embryos were assessed daily following fertilization until Day 3. Assisted hatching using Tyrode's acid was performed on embryos transferred on Day 3 . Blastocyst transfers were not performed due to the low number of oocytes retrieved per cycle. The number of embryos to be transferred was based upon embryo availability and subjective morphologic appearance of the embryos (including blastomere size, fragmentation, multinucleation). Cycle cancellation was determined upon fertilization failure or arrest of embryo development after VOR.

\section{Determination of clinical pregnancy}

Pregnancy test was performed on day 11 following embryo transfer through assessment of quantitative beta-hCG. Clinical pregnancy was defined as the presence of an intrauterine gestational $\mathrm{sac}$ on transvaginal ultrasound 21 days after embryo transfer. Pregnancy loss was defined as declining quantitative beta-hCG after the diagnosis of clinical pregnancy was made.

Statistical analysis

IVF cycles were analyzed based on insemination technique (conventional or ICSI). Data was analyzed using Analyze It $^{\circledR}$ Microsoft Excel software. Statistical analysis of continuous variables was performed using Kruskall Wallis and one-way analysis of variance (ANOVA). Categorical data was analyzed using the chi-square test. Statistical significance was defined as $P<0.05$. 


\section{Results}

Conventional insemination was used for fertilization in 179 cycles and ICSI was performed in 171 cases. Patient demographics were similar between both groups, with a mean age of 39.1 and 39.6, respectively. Additionally, baseline FSH levels were not different (12.1vs $12.4 \mathrm{IU} / \mathrm{L})$. Peak estradiol levels and the number of retrieved oocytes were not different between the two insemination methods. Fertilization rates per oocyte retrieved for CI and ICSI were comparable $(51.5 \%$ vs. $51.8 \%)$. The number of embryos available for transfer per patient was 1.8 and 1.7 respectively. Parallel implantation rates $(22 \%$ vs. $25 \%)$, clinical pregnancy rates $(32.8 \%$ vs. $33.3 \%)$, and loss rates $(26.7 \%$ vs. $39.5 \%)$ were also noted. The number of cancelled cycles prior to embryo transfer was not different (Table 1).

In our series, failure to fertilize occurred in $16.2 \%$ (29/ $179)$ and $10.5 \%$ (18/171) of CI and ICSI cases respectively $(p=0.161)$. The immaturity rate of day 0 oocytes allocated to ICSI was $23.4 \%(133 / 169)$. The immaturity rate of oocytes allocated to CI was unknown until day 1 , which was noted to be $7.2 \%(42 / 587)$. Fertilization rates of only mature oocytes allocated to CI or ICSI were $55.4 \%$ (302/ $545)$ and $67.7 \%(295 / 436(p=0.001)$. The fact that conventionally inseminated oocytes are not assessed until day 1 of culture, may explain the relatively low immaturity rate, allowing immature aspirated oocytes to mature overnight.

We further analyzed whether a difference in outcome would be noted based on specific number of oocytes aspirated. Tables 2 and 3 describe the cases by number of oocytes retrieved and outcome related to clinical pregnancy and cancellation rates, respectively. No differences were noted for these variables regardless of number of aspirated oocytes (Table 3).

\section{Discussion}

Treatment of the "poor responder" has become an enormous challenge in our field. A variety of protocols have been applied for $\mathrm{COH}$ to try to enhance response for women known to be poor responders. Micromanipulation techniques, such as assisted hatching, have also been clinically applied in this subset of patients in hope of enhancing implantation rates. Previous studies have demonstrated higher fertilization rate and improved outcomes after ICSI procedures [18]. The objective of this study was to determine if patients with a poor response to gonadotropin stimulation benefit from undergoing ICSI.

Poor response to $\mathrm{COH}$ with low oocyte retrieval has an estimated prevalence of $9-24 \%$ of IVF patients. Though definitions of poor response vary with regard to number of oocytes retrieved, many studies have suggested that low responders have lower fertilization rates, pregnancy rates, and implantation rates after an IVF cycle [13, 19]. Complete fertilization failure, which occurs in approximately $16 \%$ of IVF cycles, was significantly more frequent in cycles that resulted in 3 or fewer oocytes $[14,20]$. Though it has been suggested that 6-10 oocytes is the ideal quantity to optimize the number of embryos ultimately transferred [21], retrospective analyses of poor response cycles and subsequent fertilization and pregnancy rates suggest that embryo transfer is still possible in this patient population and cycles should not be cancelled [19, 22, 23]. While there have been prospective trials to compare IVF outcomes in CI versus ICSI, women with poor ovarian response [24] or previous low responders [25] have had cycles cancelled or have been excluded. In couples without poor response to $\mathrm{COH}$, ICSI has no proven benefit over CI and is a more invasive and expensive technique [24-28]. Prospective randomized trials studying low response to $\mathrm{COH}$ have not been performed.
Table 1 Demographics and clinical outcome of CI and ICSI cases

${ }^{\mathrm{a}} \mathrm{CI}$ conventional insemination

${ }^{\mathrm{b}}$ ICSI intracytoplasmic sperm injection

${ }^{\mathrm{c}} \mathrm{FSH}$ follicle stimulating hormone

${ }^{\mathrm{d}}$ E2 estradiol

${ }^{\mathrm{e}}$ ET embryo transfer

${ }^{\mathrm{f}}$ Kruskall-Wallis and one-way ANOVA used for continuous variables; chi-square test for categorical variables

\begin{tabular}{lccc}
\hline & $\mathrm{CI}^{\mathrm{a}}(n=179)$ & $\operatorname{ICSI}^{\mathrm{b}}(n=171)$ & $p$ value $^{\mathrm{f}}$ \\
\hline Age (yrs) & $39.1 \pm 4.1$ & $39.6 \pm 3.6$ & 0.182 \\
$\mathrm{FSH}^{\mathrm{c}}(\mathrm{IU} / \mathrm{L})$ & $12.1 \pm 6.8$ & $12.4 \pm 6.8$ & 0.645 \\
Peak E2 $^{\mathrm{d}}(\mathrm{pg} / \mathrm{mL})$ & $1092.3 \pm 512$ & $1017.8 \pm 432$ & 0.144 \\
Oocytes retrieved (n) & $3.3 \pm 0.9$ & $3.3 \pm 0.8$ & 0.598 \\
Embryos transferred (n) & $1.8 \pm 0.8$ & $1.7 \pm 0.8$ & 0.339 \\
Fertilization rate & $51.5 \%(302 / 587)$ & $51.8 \%(295 / 569)$ & 0.915 \\
Implantation rate & $22 \%(54 / 246)$ & $25 \%(54 / 219)$ & 0.502 \\
Cancellation rate & $23.5 \%(42 / 179)$ & $24.6 \%(42 / 171)$ & 0.908 \\
Clinical pregnancy rate/ET & $32.8 \%(45 / 137)$ & $33.3 \%(43 / 129)$ & 0.963 \\
Loss rate & $26.7 \%(12 / 45)$ & $39.5 \%(17 / 43)$ & 0.291 \\
\hline
\end{tabular}


Table 2 Number of CI and ICSI cases based on oocytes retrieved

\begin{tabular}{lll}
\hline Cases & Conventional insemination & $\mathrm{ICSI}^{\mathrm{a}}$ \\
\hline 1 oocyte & 7 & 4 \\
2 oocytes & 29 & 28 \\
3 oocytes & 50 & 47 \\
4 oocytes & 93 & 92 \\
TOTAL & 179 & 171 \\
\hline
\end{tabular}

${ }^{\mathrm{a}}$ ICSI intracytoplasmic sperm injection

Staessen et al. used sibling oocytes for comparison of CI and ICSI and did not demonstrate a significant difference between these techniques in terms of cleavage or embryo quality, though their study was limited by sample size [18]. In fact, Taylor et al. demonstrated a significantly higher fertilization rate with CI when compared to ICSI (53.2\% vs. $41.1 \%, p<0.0001)$ in sibling oocytes [7]. On the other hand, a variety of studies comparing ICSI with CI suggest higher fertilization rates and pregnancy outcomes with this more complex assisted reproductive technology $[4,15,16$, 18, 29]. Recently, Ou et al. published a case-control study that showed significantly higher fertilization rates for ICSI in low response cycles and a favorable trend for ICSI in clinical pregnancy rate, implantation rate and live birth rate [23]. This study, however, was limited by small sample size. Many other retrospective analyses of low response to $\mathrm{COH}$ have shown no benefit to using ICSI over CI in low responder patients with fertile sperm [20, 22, 30]. Our study demonstrates overall parallel clinical outcome, with similar fertilization rates, number of embryos available for transfer, clinical pregnancy and cancellation rates between both groups.

Decreased incidence of total fertilization failure has been described after ICSI. This result is due to ensured sperm entry into the oocyte, thereby bypassing the zona pellucida and acrosome reaction necessary for in vivo fertilization or CI $[4,16,31]$. On the other hand, others have theorized that using ICSI may bypass natural selection methods and choose abnormal sperm, resulting in poor outcomes. In our analysis, total fertilization failure was not lower in oocytes allocated to ICSI.

Maturation of the oocyte is proportional to the number that will ultimately fertilize [32]; at least $1 \mathrm{~h}$ is needed after polar body extrusion for appropriate oocyte maturation and development [33]. ICSI is limited by the oocyte maturity required for injection, whereas CI may allow immature oocytes to complete maturation in culture while awaiting fertilization. It would seem, therefore, that ICSI would be more limiting in patients with low response, given the probability that any oocytes retrieved would be within the metaphase II window of maturity for this procedure. In our series, the immaturity rate of day 0 oocytes allocated to ICSI was $23.4 \%$. The immaturity rate of oocytes allocated to $\mathrm{CI}$ was unknown until day 1 , which was noted to be $7.2 \%$. When analyzing fertilization rates of only mature oocytes allocated to CI or ICSI we encountered a significant difference between both groups (55.4\% vs. $67.7 \%)(p=0.001)$. This may be explained by the fact that a significantly higher number of immature oocytes are discarded and not allocated to undergo ICSI, after hyaluronidase exposure. We further analyzed whether a difference in clinical pregnancy rate and cancellation rates would be noted based on the specific number of retrieved oocytes. We did not encounter a significant difference in these outcomes regardless of the number of retrieved oocytes.

There is a lack of randomized controlled trials studying ICSI in poor response cycles. Clinical management has been predominantly shaped by anecdotal reports and retrospective analyses of series notable for low sample size. The objective of this analysis was to determine if patients with low response to gonadotropin stimulation benefit from the use of ICSI for insemination. Based on our results, we conclude that couples with normal semen parameters do not require that ICSI be performed for oocyte number alone. Any potential benefit of "assisting" fertilization may be outweighed by stripping oocytes of

Table 3 Clinical pregnancy per transfer and cancellation rate by oocytes retrieved

\begin{tabular}{|c|c|c|c|c|c|c|}
\hline \multirow[t]{2}{*}{ Number of oocytes } & \multicolumn{3}{|c|}{ Clinical pregnancy/ET ${ }^{\mathrm{a}}$} & \multicolumn{3}{|l|}{ Cancellation } \\
\hline & $\mathrm{CI}^{\mathrm{b}}$ & $\mathrm{ICSI}^{\mathrm{c}}$ & $p$-value ${ }^{\mathrm{d}}$ & $\mathrm{CI}^{\mathrm{b}}$ & $\mathrm{ICSI}^{\mathrm{c}}$ & $p$-value ${ }^{\mathrm{d}}$ \\
\hline $1-2$ oocytes & $30 \%(7 / 23)$ & $37 \%(7 / 19)$ & 0.8144 & $36 \%(13 / 36)$ & $41 \%(13 / 32)$ & 0.6933 \\
\hline 3 oocytes & $41 \%(15 / 37)$ & $26 \%(10 / 37)$ & 0.2525 & $26 \%(13 / 50)$ & $21 \%(10 / 47)$ & 0.8651 \\
\hline 4 oocytes & $29 \%(22 / 75)$ & $36 \%(27 / 76)$ & 0.5229 & $19 \%(18 / 93)$ & $17 \%(16 / 92)$ & 0.8769 \\
\hline
\end{tabular}

\footnotetext{
${ }^{\mathrm{a}} E T$ embryo transfer

${ }^{\mathrm{b}} C I$ conventional insemination

${ }^{\mathrm{c}}$ ICSI intracytoplasmic sperm injection

${ }^{\mathrm{d}}$ Chi-square test performed for statistical analysis
} 
their cumulus complex prematurely and preventing normal fertilization of late maturing oocytes.

Disclosures The authors report no financial conflicts of interest or financial support for this research.

\section{References}

1. Palermo GD, Cohen J, Alikani M, Adler A, Rosenwaks Z. Intracytoplasmic sperm injection: a novel treatment for all forms of male factor infertility. Fertil Steril. 1995;63:1231-40.

2. Sherins RJ, Thorsell LP, Dorfmann A, Dennison-Lagos L, Calvo LP, Krysa L, et al. Intracytoplasmic sperm injection facilitates fertilization even in the most severe forms of male infertility: pregnancy outcome correlates with maternal age and number of eggs available. Fertil Steril. 1995;64:369-75.

3. Tarlatzis BC, Bili H. Intracytoplasmic sperm injection. Survey of world results. Ann N Y Acad Sci. 2000;900:336-44.

4. Fishel S, Aslam I, Lisi F, Rinaldi L, Timson J, Jacobson M, et al. Should ICSI be the treatment of choice for all cases of in-vitro conception? Hum Reprod. 2000;15(6):1278-83.

5. Devroey P, Liu J, Nagy Z, Tournaye H, Silber SJ, Van Steirteghem AC. Normal fertilization of human oocytes after testicular sperm extraction and intracytoplasmic sperm injection. Fertil Steril. 1994;62(3):639-41.

6. Nagy ZP, Liu J, Joris H, Verheyen G, Tournaye H, Camus M, et al. The result of intracytoplasmic sperm injection is not related to any of the three basic sperm parameters. Hum Reprod. 1995;10(5):1123-9.

7. Silber SJ, Van Steirteghem AC, Liu J, Nagy Z, Tournaye H, Devroey P. High fertilization and pregnancy rate after intracytoplasmic sperm injection with spermatozoa obtained from testicle biopsy. Hum Reprod. 1995;10(1):148-52.

8. Taylor TH, Wright G, Jones-Colon S, Mitchell-Leef D, Kort HI, Nagy ZP. Comparison of ICSI and conventional IVF in patients with increased oocyte immaturity. Reprod Biomed Online. 2008;17(1):46-52.

9. Ubaldi F, Liu J, Nagy Z, Tournaye H, Camus M, Van Steirteghem $\mathrm{A}$, et al. Indications for and results of intracytoplasmic sperm injection (ICSI). Int J Androl. 1995;18 Suppl 2:88-90.

10. Nagy ZP, Verheyen G, Tournaye H, Van Steirteghem AC. Special applications of intracytoplasmic sperm injection: the influence of sperm count, motility, morphology, source and sperm antibody on the outcome of ICSI. Hum Reprod. 1998;13 Suppl 1:143-54.

11. Ruiz A, Remohí J, Minguez Y, Guanes PP, Simón C, Pellicer A. The role of in vitro fertilization and intracytoplasmic sperm injection in couples with unexplained infertility after failed intrauterine insemination. Fertil Steril. 1997;68(1):171-3.

12. Jenkins JM, Davies DW, Devonport H, Anthony FW, Gadd SC, Watson RH, et al. Comparison of 'poor' responders with 'good' responders using a standard buserelin/human menopausal gonadotrophin regime for in-vitro fertilization. Hum Reprod. 1991;6(7):918-21.

13. Dor J, Seidman DS, Ben-Shlomo I, Levran D, Karasik A, Mashiach $\mathrm{S}$. The prognostic importance of the number of oocytes retrieved and estradiol levels in poor and normal responders in in vitro fertilization (IVF) treatment. J Assist Reprod Genet. 1992;9(3):228-32.

14. Barlow P, Englert Y, Puissant F, Lejeune B, Delvigne A, Van Rysselberge M, et al. Fertilization failure in IVF: why and what next? Hum Reprod. 1990;5(4):451-6.

15. Khamsi F, Yavas Y, Roberge S, Wong JC, Lacanna IC, Endman M. Intracytoplasmic sperm injection increased fertilization and goodquality embryo formation in patients with non-male factor indications for in vitro fertilization: a prospective randomized study. Fertil Steril. 2001;72(2):342-7.
16. Orief Y, Dafopoulos K, Al-Hassani S. Should ICSI be used in nonmale factor infertility? Reprod Biomed Online. 2004;9(3):348-56.

17. Ludwig M, Strik D, Al-Hasani S, Diedrich K. No transfer in a planned ICSI cycle: we cannot overcome some basic rules of human reproduction. Eur J Obstet Gynecol Reprod Biol. 1999;87(1):3-11.

18. Staessen C, Camus M, Clasen K, De Vos A, Van Steirteghem A. Conventional in-vitro fertilization versus intracytoplasmic sperm injection in sibling oocytes from couples with tubal infertility and normozoospermic men. Hum Reprod. 1999;14(10):2474-9.

19. Jenkins JM, Davies DW, Devonport H, Anthony FW, Gadd SC, Watson RH, et al. Comparison of 'poor' responders with 'good' responders using a standard buserelin/human menopausal gonadotrophin regime for in-vitro fertilization. Hum Reprod. 1991;6(7):918-21.

20. Ludwig M, Al-Hasani S, Küpker W, Bauer O, Diedrich K. A new indication for an intracytoplasmic sperm injection procedure outside the cases of severe male factor infertility. Eur J Ob Gyn. 1997;75:207-10.

21. Melie NA, Adeniyi OA, Igbineweka OM, Ajayi RJ. Predictive value of the number of oocytes retrieved at ultrasound-directed follicular aspiration with regard to fertilization rates and pregnancy outcome in intracytoplasmic sperm injection treatment cycles. Fertil Steril. 2003;80(6):1376-9.

22. Moreno C, Ruiz A, Simon C, Pellicer A, Remohi J. Intracytoplasmic sperm injection as a routine indication in low responder patients. Hum Reprod. 1998;13:2126-9.

23. Ou YC, Lan KC, Huang FJ, Kung FT, Lan TH, Chang SY. Comparison of in vitro fertilization versus intracytoplasmic sperm injection in extremely low oocyte retrieval cycles. Fertil Steril. 2010;93(1):96-100.

24. Foong SG, Fleetham JA, O'Keane JA, Scott SG, Tough SC, Greene CA. A prospective randomized trial of conventional in vitro fertilization versus intracytoplasmic sperm injection in unexplained fertility. J Assist Reprod Genet. 2006;23(3):137-40.

25. Bhattacharya S, Hamilton MPR, Shaaban M, Khalaf Y, Seddler M, Ghobara $\mathrm{T}$, et al. Conventional in vitro fertilisation versus intracytoplasmic sperm injection for the treatment of non-male factor infertility: a randomised controlled trial. Lancet. 2001;357:2075-9.

26. Van Rumste M, Evers JLH, Farquhar C. Intra-cytoplasmic sperm injection versus conventional techniques for oocyte insemination during in vitro fertilisation in patients with non-male subfertility. Cochrane Database Syst Rev. 2003;(2) Art. No.: CD001301.

27. Van Rumste MME, Evers JLH, Farquhar CM. ICSI versus conventional techniques for oocyte insemination during IVF in patients with non-male factor subfertility: a Cochrane review. Hum Reprod. 2004;19(2):223-7.

28. Kim HH, Bundorf MK, Behr B, McCallum SW. Use and outcomes of intracytoplasmic sperm injection for non-male factor infertility. Fertil Steril. 2007;88(3):622-8.

29. Verheyen G, Tournaye H, Staessen C, De Vos A, Vandervorst M, Van Steirteghem A. Controlled comparison of conventional invitro fertilization and intracytoplasmic sperm injection in patients with asthenozoospermia. Hum Reprod. 1999;14(9):2313-9.

30. Gozlan I, Dor A, Farber B, Meirow D, Feinstein S, Levron J. Comparing intracytoplasmic sperm injection and in vitro fertilization in patients with single oocyte retrieval. Fertil Steril. 2007;87 (3):515-8.

31. Plachot M, Belaisch-Allart J, Mayenga JM, Chouraqui A, Tesquier L, Serkine AM. Outcome of conventional IVF and ICSI on sibling oocytes in mild male factor infertility. Hum Reprod. 2002;17(2):362-9.

32. Balakier H, Sojecki A, Motamedi G, Librach C. Time-dependent capability of human oocytes for activation and pronuclear formation during metaphase II arrest. Hum Reprod. 2004;19 (4):982-7.

33. Hyun CS, Cha JH, Son WY, Yoon SH, Kim KA, Lim JH. Optimal ICSI timing after the first polar body extrusion in in vitro matured human oocytes. Hum Reprod. 2007;22(7):1991-5. 